\title{
UN TEXTO RELIGIOSO DE MEDIADOS DEL SIGLO XVI EN GUATEMALA: LA "THEOLOGIA INDORUM" DE FRAY DOMINGO DE VICO
}

\author{
Cristina Bredt-Kriszat \\ Universidad de Hamburgo. Alemania.
}

Con mi ponencia tengo la intención de presentar un texto religioso, escrito en tres lenguas mayas de las tierras altas guatemaltecas por un misionero español, entre los años 1550 y 1555, para enfocarlo desde un punto de vista todavía no considerado, en cuanto al valor etnohistórico de este documento. Primeramente aportaré datos de la biografia del autor, fray Domingo de Vico, y la época en que viviera. A continuación pasaré a describir su obra: la Theologia Indorum. Luego me concentraré en el texto traducido y comentado por Vico. En base a dos ejemplos importantes señalaré la influencia ejercida por esta obra durante los siglos siguientes, no sólo sobre escritos de cronistas españoles, sino también sobre documentos de autoría indígena.

\section{FRAY DOMINGO DE VICO Y SU EPOCA}

La Iglesia guatemalteca siguió, desde la conquista (1524) hasta mediados del siglo XVI, una política propia en el empleo de las lenguas nativas [García-Ruiz 1992:87]. En poco tiempo se comprobó que el éxito en la conversión de los indígenas, dependía del grado en que cada sacerdote aprendiera las lenguas de su región a misionar.

La corona española se interesó desde un principio por lograr una evangelización más eficaz en lengua castellana [García-Ruiz 1992:86]. 
Debido al empleo de los idiomas aborígenes, se inicia la discusión sobre los criterios para traducir textos religiosos, como las Sagradas Escrituras', que concluirá al intervenir Francisco Marroquín, obispo de Guatemala [García-Ruiz 1992:94].

El aprendizaje de los idiomas nativos contaba de dos etapas. En la primera, los padres, ayudados por indígenas, traducían cantos y rezos, para posteriormente, en la segunda etapa, aprender el idioma de cada país.

$\mathrm{El}$ resultado de años de trabajo intensivo con la nueva lengua se refleja en diferentes tipos de textos, cuyo objetivo era el facilitar la labor de la próxima generación evangelizadora.

Dentro de las diferentes obras escritas por los sacerdotes, en lenguas indígenas, se pueden distinguir dos grupos principales de textos: 1) los relacionados con el idioma, y 2) los relacionados con la religión cristiana.

$\mathrm{Al}$ primer grupo de textos relacionados con el idioma pertenecen los diferentes Artes y Vocabularios, en donde la religión juega un aspecto secundario. Sólo en los ejemplos, para el empleo de un verbo o sustantivo determinado, extraídos de la vida cotidiana de un religioso, se manifiesta el origen y la intención del autor, así como quienes eran sus lectores (obra de misionero para misioneros).

Al segundo grupo, el de los textos relacionados con la religión, corresponden las diferentes: doctrinas, catecismos, tratados sobre el dogma, rezos, sermones y traducciones de las Sagradas Escrituras, como lo es el documento aquí tratado, la Theologia Indorum ${ }^{2}$ de fray Domingo de Vico.

\section{EL AUTOR}

Fray Domingo de Vico era originario de Jaén. Se desconoce la fecha exacta de su nacimiento, pero se calcula aproximadamente en 1519 [René Acuña 1985:281].

A fray Domingo se lo describe en las crónicas como:

[...] pequeño de cuerpo, aunque abultado de carnes. De un ánimo tan grande que parecia haber nacido para emperador [...] No sabía escribir en papel pequenio, ni con pluma corta [Remesal 1964 I:296].

Hizo sus estudios teológicos en Ubeda y en el convento de Santo Domingo de Salamanca. En 1545 se integra al grupo de dominicos, quienes bajo las órdenes del nuevo obispo de Chiapas, Bartolomé de las Casas, inician el viaje a las Indias. En su Historia general..., escrita entre los años 1615 y 1617, fray Antonio de Remesal [1964 y 1966] dedica algunos capítulos a 
fray Domingo, detallando en especial sus últimos años de vida. Vico se desempeñará durante dos años [Remesal 1966 II:297] como prior del convento de Santo Domingo en la Ciudad de Guatemala, luego ocupará ese mismo cargo en el convento de Cobán.

En esta última etapa de su vida, se dedica a misionar la región de la Verapaz, hasta el día de su muerte, acaecida el 29 de noviembre de 1555 durante un levantamiento de acaláes y lacandones.

Durante los diez años de estadía en Guatemala, aprendió siete idiomas indigenas. Así consta en una carta escrita por los dominicos dirigida a la Audiencia de los Confines del 14 de mayo de 1556 [AGI 1556], medio año después de fallecer Vico. Robert Carmack [1973:114-5] menciona entre estas lenguas: cakchiquel, quiché, tzutuhil, kekchí, chol y pocomán. Benno Biermann [1964:128], por su parte, añade a la lista el poconchí.

Vico escribió una gran cantidad de obras en estas lenguas, y, como menciona Remesal[1966 II:298]:

[...] que muy sin hipérbole se podía comparar lo que el padre fray Domingo de Vico había escrito en lengua de indios, a lo que Santo Tomás escribió en latín.

Si bien las obras alcanzaron una gran difusión después de su muerte, hoy en día se encuentran sólo pocos manuscritos de su autorfa ${ }^{3}$ : algunos sermones, un vocabulario y diferentes ejemplares de la denominada Theologia Indorum.

\section{LA THEOLOGIA INDORUM}

La Theologia Indorum de fray Domingo de Vico es una traducción de textos bíblicos del Antiguo y Nuevo Testamento, que fueran seleccionados y comentados por el mismo autor. Fue redactada - de acuerdo con las copias hoy día existentes - en tres idiomas nativos de Guatemala: quiché, cakchiquel y tzutuhil (ver TABLA 1).

Vico debe haberla escrito durante los 465 últimos años de su vida, después de haber sido nombrado prior del convento dominico en la capital guatemalteca (1551), zona de habla cakchiquel, lo cual debe haberle permitido un contacto intensivo con el idioma ${ }^{4}$.

En los manuscritos parisinos Fond Amér. Nros. 5 y 10, y en el de la American Philosophical Society, se menciona febrero del año 1553 como dato del original. 
TABLA 1

Manuscritos existentes de la Theologia Indorums

\begin{tabular}{|c|c|c|c|c|}
\hline Weeks $^{6}$ & $\begin{array}{l}\text { BNP' (Fond } \\
\text { Américain) }\end{array}$ & Año & Idioma & $\begin{array}{l}\text { Páginas/ } \\
\text { Folios }^{8}\end{array}$ \\
\hline $\begin{array}{l}376^{\circ} \\
676 \mathrm{PUL}^{10}\end{array}$ & $\begin{array}{l}\mathrm{N} 42 \\
-\end{array}$ & $\begin{array}{l}1675 \\
\text { s.XVI }\end{array}$ & $\begin{array}{l}\text { cakchiq. } \\
\text { cak/qui. }\end{array}$ & $\begin{array}{l}180 \mathrm{f} \\
476 \mathrm{p}\end{array}$ \\
\hline 677 PUL & - & s.XVI? & quiché & $40 p$ \\
\hline 678 PUL & - & s.XVI? & quiché & $296 \mathrm{f}$ \\
\hline $679^{11}$ & - & s.XVI? & quiché & $462 p$ \\
\hline 680 PUL & - & s.XVI? & quiché & $199 \mathrm{f}$ \\
\hline 714 & N 3 & s.XVI? & cakchig. & $275 \mathrm{f}$ \\
\hline $715^{12}$ & - & s.XVI? & cakchiq. & $448 \mathrm{f}$ \\
\hline $716^{13}$ & - & s.XVI? & cakchiq. & $271 p$ \\
\hline 717 PUL & - & s.XVI? & quiché & $25 f$ \\
\hline 718 PUL & - & s.XVI? & quiché & $505 \mathrm{f}$ \\
\hline $719^{14}$ & - & s.XVI? & quiché & $412 p$ \\
\hline 720 PUL & - & s.XVI? & quiché & $288 \mathrm{p}$ \\
\hline 721 PUL & - & 1769 & quiché & $286 p$ \\
\hline 722 PUL & - & s.XVI? & quiché & $688 p$ \\
\hline 723 & N 10 & 1553 & quiché & $101 \mathrm{f}$ \\
\hline \multirow[t]{2}{*}{724 APS 15} & - & $1553 /$ & & \\
\hline & & 1605 & quiché 16 & $185 \mathrm{f}$ \\
\hline $725^{17}$ & N 4 & s.XVI? & cakch. $^{18}$ & $185 \mathrm{f}$ \\
\hline \multirow[t]{3}{*}{$727^{19}$} & N 5 & $1553 /$ & & \\
\hline & & 1605 & quiché & $185 \mathrm{f}$ \\
\hline & $N 56^{20}$ & s.XVI? & quiché & $32 f$ \\
\hline
\end{tabular}

Durante el siglo XVIII, la Theologia Indorum era todavía leída en "Cobán y Xocopilas" [Solano 1963:330].

La cantidad de dos ejemplares resulta mínima si se la compara con el número de copias existentes en la actualidad. Francisco Ximénez, dominico español llegado a Guatemala a fines del siglo XVII, se queja de que las copias se encontraban "ocultas" en manos de "maestros y fiscales". 
La obra se compone de dos tomos o partes, y Ximénez [1977:62] las describe de la siguiente manera:

[...] la una [Antiguo Testamento], que empieza desde el ser de Dios y la creación del mundo hasta la venida de Cristo y la segunda [Nuevo Testamento] desde santa Ana y san Joaquín hasta el juicio final [...]"

\section{VICO Y SU TRADUCCIÓN COMENTADA DE LA BIBLIA}

Fray Domingo de Vico en su traducción comentada de las Sagradas Escrituras, elige ciertos libros y capítulos, mientras descarta otros. Su traducción es sin embargo en algunos pasajes de gran exactitud. Versículo por versículo pueden ser identificadas sus frases en el texto bíblico original ${ }^{21}$ (ver TABLA 2).

Al mismo tiempo Vico aprovecha términos o ideas que puedan prestarse a confusión, para aclararlos desde el punto de vista dogmático $\mathrm{y}$, a veces, entablar relación con temas del Nuevo Testamento. Los criterios seguidos por Vico, para incluir o descartar libros y capítulos bíblicos, no pueden ser precisados en toda su complejidad. Para ello, no sólo haría falta analizar la discusión a cerca de las Sagradas Escrituras durante el siglo XVI. También sería imprescindible comparar entre sí las copias de la Theologia Indorum existentes, sin olvidar obras similares en otras lenguas indígenas, para poder determinar si el autor sigue un concepto general marcado por las autoridades ecclesiásticas de la región, o si junto con la traducción del texto bíblico, transmitió su propia visión evangelizadora.

En base a dos pasajes bíblicos intentaré dar una idea de la riqueza de matices - lamentablemente ignorados - que se encuentran en la Theologia Indorum. 
TABLA 2

Correlación entre el Antiguo Testamento y la Theologia Indorum (1.a parte)

\begin{tabular}{|c|c|c|}
\hline \multicolumn{2}{|c|}{ Antiguo Testamento } & \multirow{2}{*}{$\begin{array}{l}\text { Theologia Indorum } \\
\text { Corresponde Capitulo N }\end{array}$} \\
\hline Libro & Total Capitulos & \\
\hline Génesis & 50 & $30--59^{22}$ \\
\hline Exodo & 40 & $60-71$ \\
\hline Levítico & 27 & $71--72$ \\
\hline Números & 36 & $-71-78$ \\
\hline Deuteronomio & 34 & 78 \\
\hline Josué & 24 & $79-83$ \\
\hline Jueces & 21 & 84 \\
\hline Rut & 4 & 84 \\
\hline 1 Samuel & 31 & $85-87$ \\
\hline 2 Samuel & 24 & 88 \\
\hline 1 Reyes & 22 & $88,90,91$ \\
\hline 2 Reyes & 25 & $88,90,91$ \\
\hline 1 Crónicas & 29 & -89 \\
\hline 2 Crónicas & 36 & 88,98 \\
\hline Esdras & 10 & 98 \\
\hline Nehemías & 13 & \\
\hline Toblas & 14 & 96 \\
\hline Judit & 16 & $101-2$ \\
\hline Ester & 10 & 100 \\
\hline 1 Macabeos & 16 & $-101-2$ \\
\hline 2 Macabeos & 15 & 102 \\
\hline Job & 42 & 96 \\
\hline Salmos & 150 & - \\
\hline Proverbios & 31 & - \\
\hline Eclesiastés & 12 & - \\
\hline Cantar de los Cant. & 8 & 一 \\
\hline Sabiduría & 19 & - \\
\hline Jesús, hijo Sirac & 51 & -103 \\
\hline Isaías & 66 & $-95,97-98$ \\
\hline Jeremías & 52 & -95 \\
\hline Lamentaciones & 5 & - \\
\hline Baruc & 6 & - \\
\hline Ezequiel & 48 & - \\
\hline Daniel & 14 & $92-94,101-103$ \\
\hline Oseas & 14 & - \\
\hline
\end{tabular}




$\begin{array}{lrl}\text { Joe } & 4 & - \\ \text { Amós } & 9 & - \\ \text { Abdías } & 1 & - \\ \text { Jonás } & 4 & - \\ \text { Miqueas } & 7 & - \\ \text { Nahum } & 3 & 93,-103 \\ \text { Habacuc } & 3 & - \\ \text { Sofonías } & 3 & - \\ \text { Ageo } & 2 & -95 \\ \text { Zacarías } & 14 & -\end{array}$

\section{EL PROFETA BALAM VS. RUT LA MOABITA}

Quizás no haya en toda la Theologia Indorum dos ejemplos más adecuados, para poder demostrar, tanto el alto grado de conocimiento como la sensibilidad de fray Domingo de Vico frente a las culturas indígenas de Guatemala, como lo son: los capítulos 22, 23 y 24 del cuarto libro de Moisés (Números) y el Libro de Rut.

\section{Resumen de Números, 22-24:}

Al acampar los israelitas en los campos de Moab, el rey, Balac, decide llamar al profeta Balaam para maldecir a los israelitas. Balaam le consulta a Dios, y éste le utiliza como mediador para bendecir tres veces a los israelitas. Balac se resigna, y regresa a sus dominios.

\section{Resumen de Rut, 1-4:}

Al morir su esposo, Rut opta por permanecer junto a su suegra Noemí. Ambas marchan a Belén, de donde provenía la madre de su difunto esposo. Rut se ocupa de alimentar a la anciana y así conoce a Booz, pariente de Noemí. Con ayuda de su suegra consigue que Booz la tome por esposa. De ese matrimonio nace un hijo llamado Obed.

\section{BALAM Y RUT EN LA THEOLOGLA INDORUM}

$\mathrm{Y}$ ahora cabe la pregunta: ¿đe qué manera presenta Vico estos capítulos en su traducción? 
Fray Domingo cuenta la historia de Balaam, la cual en la Biblia abarca una décima parte del Libro de los Números, en los capítulos 78 y 79 de su obra, dedicándole diez páginas (folios $117 \mathrm{v}-122 \mathrm{r}$ ). Por el contrario, la historia de Rut, que en la Biblia será contada en un libro aparte, queda, bajo la pluma de Vico ${ }^{23}$, reducida a una simple frase dentro del capítulo 84:

"Quehe ri Ruth utçilah yxok y 4oheic, quehe chi 4u ri Booz q[ue]he chi nay pu r a[l] Obeth, caib chi utçilah vinak." [Vico MS a: 136r] ${ }^{24}$

El contraste obvio en la extensión de ambas versiones (Biblia-Theologia Indorum) al reproducir las historias de Balaam y Rut, conduce obligatoriamente a la pregunta: ¿qué motivos llevaron a Vico a hacer tal diferencia entre ambos? La respuesta más simple se encuentra - dejando de lado un análisis de contenido, para el cual no alcanzaría el espacio- en el nombre del profeta: Balaam.

El nombre Rut carecía de significado para los quichés, en cambio Balam debe haber sido una especie de "déjà vu". Balam es el "tigre" [Vico MS. f:189r] americano, el jaguar, en todas las lenguas mayas. Era, además, la denominación para hechicero o "bruxa" [Smailus 1989 II:54].

Balam se usaba también, entre los quichés, como nombre adicional, para expresar una cierta cualidad de quien lo portaba [Pläschke MS.:60 y 149].

Así se llamaban tres de los cuatro primeros padres del pueblo quiché en el Popol Vuh. Sus nombres eran: Balam Quitzé, Balam Acab, Mahucutah e Iqui Balam [Recinos 1976:104]

Texto bíblico:

(Núm. 24.17) ${ }^{25}$

Lo veré, mas no ahora;

Lo miraré, mas no de cerca;

Saldrá ESTRELLA de Jacob,

Y se levantará cetro de Israel,

Y herirá las sienes de Moab,

Y destruirá a todos los hijos de Set. 


\section{Theologia Indorum:}

(folio 118r)

... xa et $\mathrm{r}$ ilic
xa et $\mathrm{v}$ mukuxic
chi 4 oheic
chi uinakiric.
nima 4 humil,
chi uae puch nima 4 hamiy
ch u xol ah israel.
qu e tçakic ah moab (...)
hunelic $x$ cha u tçih balan.
ta $x$ e r utz bijh ah israel...

(en vano su mirada, en vano su vista hacia la vida, hacia la concepción de la gran estrella. He aquí también la gran vara entre los de Israel, ellos tiran al de Moab. Para siempre dijo su palabra Balam, cuando bendijo a los de Israel.) ${ }^{26}$

\section{El Popol Vuh:}

(3 parte, capítulo IX)

Grandemente se alegraron Balam-Quitzé, Balam-Acab, Mahucutah e Iqui-Balam cuando vieron a la Estrella de la mañana. Salió primero con la faz resplandeciente, cuando salió primero delante del sol. [Recinos 1976:121]

\section{BALAM Y LA ESTRELLA}

Pero Francisco Ximénez, quien había transcrito y traducido el Popol $V u h$, y a la vez también conocía la Theologia Indorum, en la dedicatoria de su Primera parte del Tesoro de las Lenguas..., observa una coincidencia aun mayor entre el Balam bíblico y los "Balames" quichés. Ambos se ven unidos no sólo por llevar el mismo nombre, sino por anunciar o esperar una señal celestial:

Aquella estrella que esperaban, anuncio del nuevo sol, claramente la tenía profetizada el profeta Balam, y si se repara bien en la ocurrencia: Balam se llamaba su caudillo que dicen fue el que primero vio la estrella [...] [Ximénez 1985:38-39]

La coincidencia, observada por Ximénez se explica al comparar los capítulos de Balam en la Theologia Indorum (y en la Biblia) con los capítulos sobre la creación de los primeros quichés en el Popol Vuh (ver TABLA 3).

$\mathrm{La}$ coincidencia es doble, como advirtiera Ximénez ${ }^{27}$. Vico también debe haber reconocido ese paralelo, entre un Balam que profetiza (o ve) una estrella en ambas fuentes, pues traduce la escena bíblica de la "nima 4humil" (gran estrella) [Vico MS. a:118r] con el esmero que no utilizó para el Libro de Rut. 


\section{LA THEOLOGIA INDORUM Y EL ORIGEN DE LOS QUICHES}

En la Theologia Indorum se resume el pensamiento de una época, en la cual se tenía interés de subrayar lo común entre la religión católica y las indígenas, con el fin de demostrar el origen hebreo del hombre americano. Ximénez [1857:145-146] en los Escolios a las Historias del Origen de los Indios... expresa:

[...] dice el venerable Padre Fray Domingo de Vico en el capitulo 101 de la segunda parte de su "Teologia indorum": á que estos indios descienden de las diez tribus que se perdieron de los judios, y [...] que de no ser asi [...] el demonio como tan sabio [...] les sugirió estas mentiras envueltas en muchas verdades católicas [...]

\section{LA THEOLOGIA INDORUM DESDE LA PERSPECTIVA QUICHE}

Que la Theologia Indorum haya jugado un papel importante para las posteriores generaciones de misioneros, es una consecuencia comprensible.

Pero ¿̇e qué modo reaccionaron los quichés frente a un texto como el de Balam? ¿se identificaron de una manera general con las Sagradas Escrituras, o utilizaron el texto de una forma más diferenciada?

Si uno se concentra en los manuscritos quichés tempranos, en donde tanto la tradición bública como la indígena tengan cabida, la elección adecuada recae entre los diferentes titulos de tierras.

Dos de los mismos reunen a primera vista los requisitos esperados: 1) el Titulo de Pedro Velasco [Carmack y Mondloch 1989:139], el cual data aproximadamente del año 1592, y 2) el Titulo de Totonicapán [Carmack y Mondloch 1983:11], existente en una copia del siglo XVII-XVIII, si bien el original dataría de 1554 .

Ambos se inician con relatos extraidos de la Theologia, para pasar luego a la tradición quiché. En esta segunda parte se menciona a los "primeros padres" Balam-Quitze, Balam-Acab, Mahucutah e Iqui Balam, es decir, se incluyen pasajes similares al Popol Vuh.

\section{EL TITULO DE PEDRO VELASCO}

En los dos primeros folios del Titulo de Pedro Velasco se nombra a un "primer libro" llamado "Indorum" [Carmack y Mondloch 1989:140, 144 y 173-4], en el cual se cuenta el origen de los ancestros. Los autores del titulo se refieren a los mismos, como si fueran los descendientes de Moisés y Aarón, $e$ incluyen dentro de este contexto a los antepasados clásicos: Balam-Quitzé, Balam-Acab, etc. 
En este documento se emplea el mismo argumento utilizado por los misioneros, en cuanto al origen hebreo del pueblo quiché.

Sin embargo la mención de ciertos patriarcas sólo parece ser casual. En base a este sólo título no se puede demostrar, si sus autores identificaran al Balam b́́blico como a uno de los "Balames" autóctonos.

\section{EL TITULO DE TOTONICAPÁN}

En el Titulo de Totonicapán no se menciona a la Theologia Indorum expresamente, pero los pasajes tomados de la misma se extienden desde el folio $1 \mathrm{r}$ hasta $7 \mathbf{v}$, a partir del cual se inicia la tradición quiché.

Hasta aquí nos encontramos frente a una situación similar a la del título anterior, pero si se depara en el contenido del último folio (7r), se descubre una lista de lugares (ver TABLA 4) en donde el pueblo israelita acampó, durante su búsqueda de la tierra prometida.

\section{TABLA 4}

Texto con lista de lugares bíblicos

Título de Totonicapán [Carmack y Mondloch 1983:66]:

[...] mara ubi nabe huyub

ta xuh3ax v lo3 4haka palo:

$\mathrm{v}$ cab xelimcutz:

$r$ ox sin:

$v$ cah rabitin:

r o xiney:

v ua3a3 caxerot:

$v$ uu3 chi4ates

$\checkmark$ uahxa3: eton:

v beleh: hor:

$v$ lahuh chiboch $v$

hulah chiabatin.

v 4ablah: çaret:

r oxlah arno -:

$v$ cahlah matan

r olah xchamel:

$v$ ua3lah: bemot

v uu3lah chimoab."
Mará se llama el primer cerro cuando pasamos aquí desde el otro lado del mar; el segundo fue Xelimcuts;

el tercero $\operatorname{Sin}$, el cuarto Rabiquín;

el quinto Xiney;

el sexto Caxerot;

el séptimo Chicátés;

el octavo Etón;

el noveno Jor;

el décimo Chiboch;

el undécimo Chiabatín;

el duodécimo Saret;

el décimo tercero Arnón;

el décimo cuarto Matán;

el décimo quinto Xchamel;

el décimo sexto Bemot;

el décimo séptimo Chimoab." ${ }^{28}$ 
Theologia Indorum, folio $117 \mathrm{v}$ :

4ate 4ut ta $x$ e opo r al4ual Jacob chi ri Hor [...] ta x e opon chi Obot. $x$ e el chi $4 u$ chi ri ta $x$ e opon chi Abari $x$ e el chi $4 u$ chi ri $x$ e opon chi Jared. $x$ e el chi $4 u$ chi ri $x$ e opon ch $u$ uulel Aron. $x$ e el chi $4 u$ chi ri $x$ e opo - Mathana. $x$ e el chi $4 u$ chi ri $x$ e opon Mahaliel. $x$ e el chi $4 u$ chi ri $x$ e opon Bamoth. $x$ e el chi $4 u$ chi ri Bamoth $x$ e opon Moab. ${ }^{29}$

En su nota de comentario correspondiente a este pasaje, Carmack [y Mondloch 1983:212] expresa:

[...] Nuestros autores vuelven a referir las peregrinaciones de los israelitas después de salir de Egipto. Sacan nombres mencionados en varios capítulos de la Theologia, comenzando con el sesenta y cinco. No ha sido posible localizar todos los nombres en la Theologia, en parte porque nuestros autores los modifican para conformarlos mejor al idioma quiché.

Todos estos nombres de lugares bíblicos se encuentran en la Theologia Indorum, basta sólo con tomar el primer folio del capítulo 76, para hacer un descubrimiento importante: mientras los primeros ocho nombres que aparecieran en el Título de Totonicapán, se encuentran repartidos entre los capítulos 65 hasta 75 (folios 101r-117r) de la Theologia Indorum, en el capítulo 76 (folio 117v) se pueden identificar los nueve restantes.

Si se comparan los datos de ambos documentos con los bíblicos (ver TABLA 5), se observa que en el Título de Totonicapán, como también apuntara Carmack [1983:212], algunos nombres han sido adaptados al quiché, como: Xelimcutz, 4ate, Xiney, Caxerot, Etom y Xchamel. Pero a su vez en dos casos se ha conservado una ortografía más correcta que la de la misma Theologia Indorum: Çaret y Arnón. Esto podría indicar que los autores del Titulo... habrían consultado tal vez el manuscrito original, o por lo menos una copia más fiel de la Theologia Indorum, que la utilizada para esta ponencia.

Retomando las palabras de Carmack:

Uno sólo puede imaginar la satisfacción que nuestros autores nativos sintieron al registrar como Chimoab el lugar biblico de Moab [...] [Carmack y Mondloch 1983:20]

Porque este "...suena como una palabra quiché..."

De la misma manera se puede uno imaginar el inmenso regocijo que les produjera encontrar un nombre como el de Balam en el texto bíblico. 
TABLA 5

Comparación lista de lugares bíblicos

(Exodo 15.23 - Números 21.20)

\begin{tabular}{lll}
\hline Título de Totonicapán & Theologia Indorum & Biblia \\
\hline mara & Mara & Mara \\
xelimcutz & Elim & Elim \\
sin & Sin & Sin \\
rabitin & Raphitin & Refidim \\
xiney & Monte Sinay & Monte Sinaí \\
caxerot & Serot & Hazerot \\
4ates & Cates & Cades \\
eton & Edom & Edom \\
hor & Hor & Hor \\
boch & Obot & Obot \\
abatin & Abari- & Ije-abarim \\
çaret & Jared & Zered \\
arnor & Aron & Arnón \\
matan & Mathana & Matana \\
xchamel & Mahaliel & Nahaliel \\
bemot & Bamoth & Bamot \\
chimoab & Moab & Moab \\
\hline
\end{tabular}

La prueba de ello se esconde en la lista de lugares presentada.

Los autores del título no hacen un simple resumen de la Theologia Indorum. Los pasajes escojidos provienen todos del Antiguo Testamento, para ser más exacta, del Pentateuco. Con el cuarto libro de Moisés, Números, termina la tradición bíblica en el Título de Totonicapán. Pero esta decisión, de concluir la narración abruptamente, no es producto de la casualidad, o del paulatino desinterés de sus autores por los temas abordados. Justamente alli, en esa enumeración, se encuentra la clave: la lista de nombres, entre Hor y Moab, precede en la Theologia - y por ende en la Biblia - a la historia del profeta Balaam de los moabitas. Esa misma lista antecede en el Título de Totonicapán el inicio de mitología quiché ${ }^{30}$.

El Balaam bíblico no tiene cabida en el título. Su lugar lo asumen los "Balames" nativos. Pero su presencia en la Theologia, debe haber servido a los autores indígenas de señal para dar paso a su propia historia. 
En Balam se funden ambas tradiciones. Con Balam se puede aclarar de una manera aun más eficiente -y comprender- la suposición del origen hebreo de los indígenas, $\mathrm{y}$ en especial de los quichés.

\section{LA THEOLOGLA INDORUM Y EL ESTUDIO DE TEXTOS RELIGIOSOS EN LENGUAS INDIGENAS}

Quien estudia lenguas indígenas coloniales, en este caso quiché, concentra su labor sobre documentos de garantizada autoría nativa. Bastaría con mencionar las diversas publicaciones sobre el Popol Vuh, o las ediciones críticas de algunos titulos, etc.

Entre las obras escritas por misioneros, sólo se recurre a gramáticas o vocabularios, por ser las únicas fuentes que permiten una reconstrucción del idioma indígena hablado a principios de la colonia. Se pueden mencionar por ejemplo: el Arte de la lengua 4íché o utlatecat... de fray Domingo de Vico [MS f] ${ }^{31}$, su Vocabulario de la lengua cakchiquel... Quiché y Tzutubil... [MS e] ${ }^{32}$ o el Vocabulario en Lengua Castellana y guatemalteca que se llama Cakchiquel Chi [Smailus 1989].

Pocos se interesan por manuscritos, cuyo contenido aparenta ser puramente cristiano, sobre todo si su autor era un sacerdote. El desinterés por dichos textos se debe a diferentes razones, entre las cuales cuentan:

- el empleo de un idioma elemental, debido a que el autor es extranjero ${ }^{33}$,

- la carencia de contenidos precolombinos,

- la reiteración de contenidos "europeos" conocidos, etc.

En el caso concreto de la Theologia Indorum existen pocos artículos sobre la misma.

Günther Zimmermann y Berthold Riese [1980] presentan una traducción del capítulo inicial del primer tomo ${ }^{34}$.

René Acuna, en dos ${ }^{35}$ de sus artículos, se ha dedicado principalmente al capítulo 25 del primer tomo ${ }^{36}$, cuyo tema central es el culto precolombino. El autor también aprovecha para criticar la actitud cientifica al ignorar la importancia de la obra [Acuña 1985:283].

Tanto en el Titulo de Totonicapán [Carmack y Mondloch 1983], como en el Titulo de Pedro Velasco [Carmack y Mondloch 1989:139-192], se encuentran citas de la Theologia Indorum, por lo cual la misma será tratada al margen de estas ediciones. 
Como René Acuña observara, en Quichean Civilization de Robert Carmack [1973], el autor "ni siquiera juzga oportuno enumerarla [a la Theologia Indorum] entre las fuentes etnohistóricas y etnográficas que describe." [Acuña 1985:283]

\section{CONCLUSION}

Fray Domingo de Vico era un misionero con aptitudes lingǘsticas destacadas. Durante sus diez años de estadía en Guatemala tomó contacto y aprendió a reconocer lo importante, lo especial de esa cultura, y sus obras reflejan ese conocimiento. En sus escritos, al traducir temas religiosos, Vico se concentra en adaptarlos - lógicamente desde $s u$ punto de vista - a las inquietudes de la población indígena.

En el caso especial de la Theologia Indorum Vico busca elementos familiares (nombres, listas de descedencias, el éxodo, etc.), en base a ellos, y a sus conocimientos de la mitología indígena, se decide por el contenido a traducir, con el fin de lograr una coincidencia entre la información brindada y la expectativa del "lector".

Con su obra Vico brinda importantes argumentos en la discución sobre el origen de los indígenas, no sólo para los españoles. Más allá de la intensión del autor o de sus lectores, independientemente si estuvieran convencidos o no de lo escrito, los quichés incluirán esos mismos argumentos en los titulos, avalando así su origen hebreo.

En la investigación futura de documentos coloniales en lenguas indígenas serf́a necesario corregir la indiferencia existente frente a los textos religiosos. Sólo por medio de un estudio profundo de la Theologia Indorum y otros textos similares, sería posible comprender la dimensión de estas obras y a la vez se podría delimitar con mayor precisión la influencia que ejercieran sobre los documentos indígenas de la época. 


\section{NOTAS}

1 En el Indice de 1551, se "prohíbe [la lectura de] la 'Biblia en romance castellano o en cualquier otra lengua'..." [Andres 1977 II:636] que no fueran las clásicas, medida que será reiterada en la lista de libros prohibidos de 1559.

De aqui en adelante el nombre Theologia Indorum se referirá al manuscrito de la American Philosophical Sociery, Class. $497 \mathrm{~N}$ Ua 13 [Vico MS a]. En caso de consultarse otras de las copias, se las mencionará expresamente.

Brasseur de Bourbourg [1871:152] expresa a su vez: "Vico possédait admirablement les principales langues de Guatémala et écrivit, entre autres ouvrages, six grammaires de langues différentes. Ces ouvrages, restés manuscrits, disparurent les uns après les autres..."

Acufia [1985:284] supone que originariamente fue escrita en cakchiquel.

5 En esta lista no se incluyen las copias fotográficas existentes.

6 Manuscritos de la Gates-Collection, compilados por Weeks [1990].

7 Manuscritos en la Bibliothèque National de Paris, según el catálogo de Omont [1925].

8 Una " $\mathrm{p}$ " detrás de la cifra indica la paginación, una " $\mathrm{f}$ " la foliación.

9 Equivocadamente en Weeks [1992:130] se le adjudica la autorfa de esta Theologia a Francisco Maldonado, Omont [1925:14] nombra sólo a Vico.

10 Original en la Princeton University Library.

11 Ubicación desconocida [Weeks 1990:190]. Existen copias fotográficas en la Library of Congress, Washington D.C.; Newberry Library, y en la Harold B. Lee Library de la Brigham Young University.

12 Ubicación desconocida [Weeks 1990:197]. Existe una copia fotográfica en la Tozzer Library, Peabody Museum of Archaeology and Ethnology, Harvard University.

13 Ubicación desconocida [Weeks 1990:197]. Tampoco existe una copia fotográfica.

14 Ubicación desconocida [Weeks 1990:197]. Existen copias fotográficas en la Library of Congress, Washington D.C.; Newberry Library, y en la Harold B. Lee Library de la Brigham Young University.

15 Original en la American Philosophical Society en Filadelfia. Class. 497A N Ua 13.

16 Erróneamente se considera escrita en cakchiquel [Weeks 1990:198]

17 Los datos de Weeks [1990:198] no coinciden exactamente con el documento parisino, pero debe tratarse del mismo.

18 Acuña [1985:289] menciona como idioma del documento al tzutuhil.

19 Los datos de Weeks [1990:198] no coinciden exactamente con el documento parisino, pero debe tratarse del mismo.

20 El título de este documento es Algunos sermones en lengua quiché de Rabinal... [Omont 1925:18], insertado se encuentran 21 capitulos de la Theologia Indorum.

21 Como texto b́blico original entiendo la Biblia Vulgata de Gutenberg (impresa 1452-55). Gutenberg tomó como modelo a la Biblia utilizada en la Universidad de París [NCE II 1967:450]. Alll se habían formado los más destacados catedráticos españoles de la época.

22 Los números de capitulos precedidos por una tilde "-", indican que su identificación es dudosa. Igual en los documentos parisinos Fond Amér. 4, 5, 10 y 42 Vico MSS. b.

24 Traducción libre: Asi mismo Ruth, buena su vida de mujer, así Booz, aś́ también su hijo [de la madre] Obeth, ambos [eran] buenas personas.

25

Biblia 1992:161. 
Traducción libre.

Una observación similar realiza en Yucatán López Cogolludo al relatar las profecías de Chilam Balam [comunicación personal de Helga-Maria Miram].

[Carmack y Mondloch 1983:173-4]

Traducción libre: Después, cuando llegaron los hijos de Jacob ahí a Hor [...] entonces llegaron a Obot. Salieron después de ahi, entonces llegaron a Abarim. Salieron después de ahl, llegaron a Jared. Salieron después de ahl, llegaron a lo del enemigo Aron. Salieron después de ahi, llegaron a Mathana. Salieron después de ahl, llegaron a Mahaliel. Salieron después de ahi, llegaron a Bamoth. Salieron después de ahi, Bamoth, Llegaron a Moab.

El nombre Balam es irrelevante para los cakchiqueles porque se basan en una tradición diferente a la quiché.

1 Sobre la autoría del documento Brasseur de Bourbourg [1871:153] aclara: "Ce MS. m'a été donné par un chef indigène de Rabinal, en 1855. Il m'assura qu'il était du père Domingo de Vico, ce que semblerait confirmer la lettre $t$ finale du vocable Utlatecat qui s'y trouve; forme antique et entièrement inusitée depuis longtemps. C'est sur la parole de ce chef, que j'ai inscrit le nom de Vico sur ce document."

32 Se encuentra en impresión un análisis lexicológico del manuscrito, por parte de: Ursula Holl, Karin Pläschke, Ortwin Smailus, Anja Stiehler y la autora de esta ponencia.

"Es virtualmente imposible que una persona que hubiera aprendido el quiché como segundo idioma, hubiera podido conocer los complejos subtipos de los diferentes estilos de habla o los hubiera podido utilizar con la perfección que aparecen el el Popol Vuh." [Mondloch 1983:99]

Corresponde al manuscrito de la Bibliothèque National en Paris, Fond Américain 5.

Existe un tercer trabajo de Acuña del año 1979, mencionado por Mondloch [1983:99], que no ha estado a mi disposición. Por ese motivo sólo me limito a tratar los dos restantes.

Su trabajo se basa en los manuscritos Fond Américain 4, 5, 10 y 42 [Aсийа 1983:7; 1985:289].

\section{B I B LIOGRAFIA}

ACUÑNA, RENEE. 1983. El Popol Vuh, Vico y la Theologia Indorum. En: Robert M. Carmack, Francisco Morales S. (eds.) Nuevas Perspectivas sobre el Popol Vuh, mpágs. 1-16. Guatemala.

- 1985. La Theologia Indorum de fray Domingo de Vico. En: Tlalocan, vol. X, págs. 281-307, UNAM. México

AGI. 1556. Archivo General de Indias (Sevilla), exp. Guatemala, Leg, 168, 14/5/1556.

ANDRES, MELQUIADES. 1977. La teologia española en el siglo XVI. Biblioteca de Autores Cristianos 14. Tomos I y II, Madrid.

BIBLIA. 1992. La Santa Biblia. Antiguo y Nuevo Testamento. Antigua Versión de Casiodoro de Reina (1569). Sociedades Bíblicas Unidas. Corea.

BIERMANN, BENNO, O. P. 1964. Missionsgeschichte der Verapaz in Guatemala. En: Jabrbuch fuir Geschichte, Staat, Wirtschaft und Gesellschaft Lateinamerikas, Tomo 1, págs.: 117-156. Köln. 
BRASSEUR DE BOURBOURG, CHARLES ETIENNE. 1871. Bibliothique Mexico-Guatemalienne. Précédé d'un coup d'oeil sur les études américaines. Paris.

BREDT-KRISZAT, C. / U. HOLL / K. PLÄSCHKE / O. SMAILUS / A.STIEHLER, MS. Vocabulario de la Lengua Cakchiquel con advertencia de los Vocablos de las Lenguas Quiche y Tzutuhil ... Andlisis lexicologico del manuscrito Bibliothique Nationale de Paris - Fond Amer. N 46. Transcripción, introducción y apéndices [En impresión].

CARMACK, ROBERT M. 1973. Quichean civilization. The ethnohistoric, ethnographic, and archaeological sources. University of California Press, Berkeley.

CARMACK, ROBERT M. Y MONDLOCH, JAMES L. 1983. El Titulo de Totonicapdn. Texto, traducción y comentario. UNAM, México.

- 1989. El Titulo de Yax y otros documentos quichés de Totonicapán, Guatemala. Edición facsimilar, transcripción, traducción y notas de Robert M. Carmack, James L. Mondloch. UNAM, México.

GARCIA-RUIZ, JESÚS. 1992. El misionero, las lenguas mayas y la traducción. Nominalismo, tomismo y etnolingüistica en Guatemala. En: Archives de Sciences Sociales des Religions, 77 (janviermars), págs. 83-110. Paris.

MONDLOCH, JAMES. 1983. Una comparación entre los estilos de habla del quiché moderno y los encontrados en el Popol Vuh. En: Nuevas perspectivas sobre el Popol Vuh. Robert M. Carmack y Francisco Morales Santos (eds.). Guatemala.

NCE. 1967. New Catbolic Encyclopedia, Vol. II Baa to Cam. The Catholic University of America, Washington.

OMONT, H. 1925. Catalogue des manuscrits américains de la Bibliotheque Nationale. En: Revue des Bibliothéques, n 1-6, 1925. Paris.

PLÄSCHKE, KARIN. MS. Rahpop nim ha. Untersuchung zur Terminologie von Amts- und Verwandtschaftsgruppenbezeichnungen in Quellen der Quiche-Indianer. Wissenschaftliche Hausarbeit zur Erlangung des akademischzen Grades eines Magister Artrium der Universität Hamburg. Hamburg 1991.

RECINOS, ADRIÁN. 1976. Popol Vuh. Las antiguas historias del Quiché. Traducidas del texto original con introducción y notas de Adrián Recinos. Fondo de Cultura Popular, 4 edición, décima primera reimpresión. México.

REMESAL, FRAY ANTONIO DE. 1964 y 1966. Historia general de las Indias Occidentales y particular de la Gobernación de Chiapa y Guatemala. Tomos I y II. Edición de P.Carmelo Sáenz de Santa Marfa, S.J.. Biblioteca de Autores Espafioles. Madrid.

SMAILUS, ORTWIN. 1989. Vocabulario en lengua castellana y guatemalteca que se llama Cakchiquel Chi. Tomos I-III. Hamburg.

SOLANO, FRANCISCO DE. 1963. Los libros del Misionero en Guatemala (Siglo XVIII). En: Missionalia Hispanica, 20. Madrid

VICO, FRAY DOMINGO DE. MS a. Theologia Indorum. Am. Phil. Soc. Ua 13.

- MSS b. Theologia Indorum. BNP Fond Américain 3-5, 10, 42.

- MS c. Algunos sermones en lengua quiché de Rabinal BNP Fond Américain 56

- MSS d. Theologia Indorum. Firestone Library de la Universidad de Princeton, New Jersey (Weeks n 676-678, 680, 717-718, 720-722)

- MS e. Vocabulario de la Lengua Cakchiquel con advertencia de los Vocablos de las Lenguas Quiche y Tzutubil se traslado de la Obra compuesta por el llmo. Padre, el venerable Fr. Domingo de Vico. BNP Fond Américain N 46.

- MS f. Arte de la lengua giché 6 utlatecat, seguido del modo de bien vivir: en la misma lengua, de todo sacado de los escritos del ven. Padre Fr. Domingo de Vico. BNP Fond Américain 63. 
WEEKS, JOHN M. 1990. Mesoamerican Ethnohistory in United States Libraries. Reconstruction of the William E. Gates Collection of Historical and Linguistic Manuscripts. Culver City, California.

XIMÉNEZ, FRANCISCO OP. 1857. Las Historias del Origen de los Indios de esta Provincia de Guatemala, traducidas de la Lengua quiche al castellano para más comodidad de los ministros del S. Evangelio. Introducción y Anotaciones por el Dr. C. Scherzer. Viena.

- 1977. Historia de la Provincia de San Vicente de Chiapa y Guatemala de la Orden de Predicadores. Biblioteca "Goathemala", Tomo XXVIII, Libros I y II. Guatemala.

-1985. Primera parte del Tesoro de las Lenguas Cakchiquel, Quiche y Zutubil, en que las dichas lenguas se traducen a la nuestra española. Ed. crítica de Carmelo Sáenz de Santa Marfa. Academia de Geografla e Historia de Guatemala. Publicación especial No. 30. Guatemala.

ZIMMERMANN, GUUNTER Y RIESE, BERTOLD. 1980. Kapitel 1 aus Domingo de Vicos "Theologia Indorum" in der Sprache der Quiché-Indianer von Guatemala. En: Antropos, Vol. 75, págs. 612-617. 